\title{
Magnetic Moments of Dirac Neutrinos
}

\author{
Nicole F. Bell*, V. Cirigliano*, M. J. Ramsey-Musolf*, P. Vogel* and \\ Mark B. Wise* \\ ${ }^{*}$ California Institute of Technology, Pasadena, CA 91125, USA
}

\begin{abstract}
The existence of a neutrino magnetic moment implies contributions to the neutrino mass via radiative corrections. We derive model-independent "naturalness" upper bounds on the magnetic moments of Dirac neutrinos, generated by physics above the electroweak scale. The neutrino mass receives a contribution from higher order operators, which are renormalized by operators responsible for the neutrino magnetic moment. This contribution can be calculated in a model independent way. In the absence of fine-tuning, we find that current neutrino mass limits imply that $\mu_{v}<10^{-14}$ Bohr magnetons. This bound is several orders of magnitude stronger than those obtained from solar and reactor neutrino data and astrophysical observations.
\end{abstract}

Keywords: Neutrino magnetic moments, neutrino mass.

PACS: $13.15 .+\mathrm{g}, 13.40 . \mathrm{Em}, 14.60 . \mathrm{Lm}$

Current advances in uncovering the pattern of neutrino mass and mixing, lead naturally to questions about more exotic neutrino properties, such as the magnetic moment, $\mu_{v}$. In this paper, we describe how the smallness of the neutrino mass may be used to set a strong model-independent limit on the size $\mu_{v}$ [1]. Neutrino magnetic moments are reviewed in [2], and recent work can be found in [3]. In the Standard Model (SM), extended to contain right-handed neutrinos, $\mu_{v}$ is non-zero but unobservably small, $\mu_{v} \simeq 3 \times 10^{-19}\left[m_{v} / 1 \mathrm{eV}\right]$ [4]. Current limits are several orders of magnitude larger, so a magnetic moment anywhere near the present limits would certainly be an indication of physics beyond the SM. The best laboratory limits arise from neutrino-electron scattering. The weak and electromagnetic contributions to $v-e$ scattering are comparable if

$$
\frac{\mu_{v}^{\exp }}{\mu_{B}} \simeq \frac{G_{F} m_{e}}{\sqrt{2} \pi \alpha} \sqrt{m_{e} T} \sim 10^{-10} \sqrt{\frac{T}{m_{e}}},
$$

where $T$ is the kinetic energy of the recoiling electron. The present limits derived from solar and reactor neutrino experiments are $\mu_{v} \lesssim 1.5 \times 10^{-10} \mu_{B}[5]$ and $\mu_{v} \lesssim 0.9 \times$ $10^{-10} \mu_{B}[6]$ respectively. A more stringent limit can be derived from bounds on energy loss in stars, $\mu_{v} \lesssim 3 \times 10^{-12} \mu_{B}$ [7].

The presence of a non-zero neutrino magnetic moment will necessarily induce a correction to the neutrino mass term. (The problem of reconciling a large magnetic moment with a small mass has been recognized in the past, and possible methods of overcoming this restriction through the use of symmetries are discussed in [8].) Assuming that $\mu_{v}$ is generated by physics beyond the SM at a scale $\Lambda$, its leading contribution to the neutrino mass, $\delta m_{v}$, scales with $\Lambda$ as

$$
\qquad \delta m_{v} \sim \frac{\alpha}{32 \pi} \frac{\Lambda^{2}}{m_{e}} \frac{\mu_{v}}{\mu_{B}},
$$


where $\delta m_{v}$ is a contribution to $m_{v}$ arising from radiative corrections at one-loop order. The $\Lambda^{2}$ dependence arises from the quadratic divergence of the dimension four neutrino mass operator, $\mathscr{O}_{M}^{(4)} \equiv(\bar{L} \tilde{\phi}) v_{R}$. Although the precise value of this term cannot be calculated in a model-independent way, we can estimate that for $\Lambda \gtrsim 1 \mathrm{TeV}$ and $\delta m_{v} \lesssim 1 \mathrm{eV}$, we require $\mu_{v} \lesssim 10^{-14} \mu_{B}$. Given the $\Lambda^{2}$ dependence, this bound becomes considerably more stringent for $\Lambda$ well above the electroweak (EW) scale. However, if $\Lambda$ is not significantly larger that the EW scale, higher dimension operators are important, and their contribution to $m_{v}$ can be calculated in a model independent way.

We start by constructing the most general operators that could give rise to a magnetic moment operator, $\bar{v}_{L} \sigma^{\mu v} F_{\mu v} v_{R}$. Demanding invariance under the SM gauge group $\mathrm{SU}(2)_{\mathrm{L}} \times \mathrm{U}(1)_{\mathrm{Y}}$, we have the following $6 \mathrm{D}$ operators

$$
\mathscr{O}_{B}^{(6)}=\frac{g_{1}}{\Lambda^{2}} \bar{L} \tilde{\phi} \sigma^{\mu v} v_{R} B_{\mu v}, \quad \mathscr{O}_{W}^{(6)}=\frac{g_{2}}{\Lambda^{2}} \bar{L} \tau^{a} \tilde{\phi} \sigma^{\mu v} v_{R} W_{\mu v}^{a}
$$

After spontaneous symmetry breaking, both $\mathscr{O}_{B}^{(6)}$ and $\mathscr{O}_{W}^{(6)}$ contribute to the magnetic moment. Through renormalization, these operators will also generate a contribution to the $6 \mathrm{D}$ neutrino mass operator

$$
\mathscr{O}_{M}^{(6)}=\frac{1}{\Lambda^{2}} \bar{L} \tilde{\phi} v_{R}\left(\phi^{\dagger} \phi\right)
$$

The three operators, $\left\{\mathscr{O}_{B}^{(6)}, \mathscr{O}_{W}^{(6)}, \mathscr{O}_{M}^{(6)}\right\}$ constitute a closed set under renormalization, so that our effective Lagrangian is given by

$$
\mathscr{L}_{\text {eff }}=C_{B}(\mu) \mathscr{O}_{B}^{(6)}+C_{W}(\mu) \mathscr{O}_{W}^{(6)}+C_{M}(\mu) \mathscr{O}_{M}^{(6)}
$$

where the operator coefficients, $C_{i}(\mu)$, depend upon the energy scale $\mu$. The magnetic moment and mass are related to the operator coefficients as

$$
\begin{aligned}
\frac{\mu_{v}}{\mu_{B}} & =-4 \sqrt{2}\left(\frac{m_{e} v}{\Lambda^{2}}\right)\left[C_{B}(v)+C_{W}(v)\right], \\
\delta m_{v} & =-C_{M}(v) \frac{v^{3}}{2 \sqrt{2} \Lambda^{2}} .
\end{aligned}
$$

To connect $\mu_{v}$ with $m_{v}$, we thus need to find the relationship between the coefficients $C_{i}(\mu)$ at the weak scale, $\mu=v$. This requires that we solve the renormalization group equations (RGE) which relate $C_{i}(\Lambda)$ to $C_{j}(v)$.

Figures $(1,2)$ display representative examples of the one-loop diagrams which contribute to the renormalization of the 6D operators. (See Ref. [1] for further details.) Solving the RGE, retaining only the leading logarithms, we find that $\mu_{v}$ and $m_{v}$ are related as

$$
\frac{\mu_{v}}{\mu_{B}}=\frac{G_{F} m_{e}}{\sqrt{2} \pi \alpha}\left[\frac{\delta m_{v}}{\alpha \ln (\Lambda / v)}\right] \frac{32 \pi \sin ^{4} \theta_{W}}{9 f},
$$

where $\theta_{W}$ is the weak mixing angle,

$$
f=(1-r)-\frac{2}{3} r \tan ^{2} \theta_{W}-\frac{1}{3}(1+r) \tan ^{4} \theta_{W}
$$




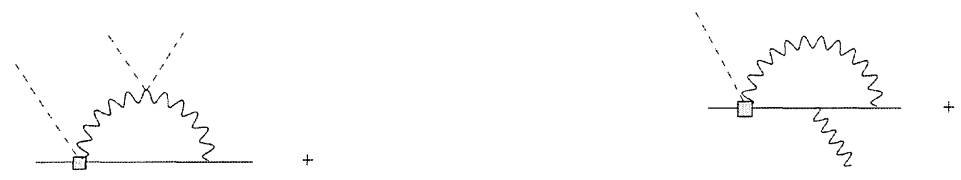

FIGURE 1. Renormalization of the mass operator, $\mathscr{O}_{M}^{(6)}$, due to insertions of $\mathscr{O}_{B, W}^{(6)}$ (left); selfrenormalization of $\mathscr{O}_{B, W}^{(6)}$ (right).
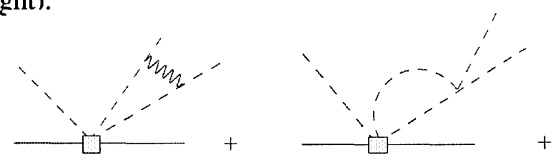

FIGURE 2. Self-renormalization of $\mathscr{O}_{M}^{(6)}$.

and $r$ is a ratio of operator coefficients at scale $\Lambda, r \equiv\left[C_{B}(\Lambda)-C_{W}(\Lambda)\right] /\left[C_{B}(\Lambda)+C_{W}(\Lambda)\right]$.

For $\Lambda \gtrsim 1 \mathrm{TeV}$, the bound becomes

$$
\frac{\mu_{v}}{\mu_{B}} \lesssim 8 \times 10^{-15} \times\left(\frac{\delta m_{v}}{1 \mathrm{eV}}\right) \frac{1}{f},
$$

and so for $f \simeq 1$ and $m_{v} \lesssim 1 \mathrm{eV}$, we find $\mu_{v} \lesssim 10^{-14} \mu_{B}$. In principle, larger values of $\mu_{v}$ could be obtained, but only by fine-tuning the coefficients $C_{i}(\Lambda)$ to arrange cancellations in Eq. 9 such that $f \ll 1$. We therefore conclude that the natural size of $\mu_{v}$ for Dirac neutrinos is at least $10^{2}$ times stronger than astrophysical limits and $10^{4}$ times stronger than reactor and solar neutrino bounds. The limits that can be placed on transition magnetic moments of Majorana neutrinos are substantially weaker than the Dirac case, and have recently been calculated in [9].

\section{ACKNOWLEDGMENTS}

This work was supported in part under U.S. DOE contracts DE-FG02-05ER41361 and DE-FG03-92ER40701, and NSF grant PHY-0071856.

\section{REFERENCES}

1. N. F. Bell et al., Phys. Rev. Lett. 95, 151802 (2005).

2. M. Fukugita and T. Yanagida, Physics of neutrinos and applications to astrophysics, Chapter 10, Springer, Berlin, (2003), and references therein; B. Kayser, F. Gibrat-Debu and F. Perrier, World Sci. Lect. Notes Phys. 25, 1 (1989); H. T. Wong and H. B. Li, Mod. Phys. Lett. A 20, 1103 (2005).

3. G. C. McLaughlin and J. N. Ng, Phys. Lett. B 470, 157 (1999); R. N. Mohapatra, S. P. Ng and H. b. Yu, Phys. Rev. D 70, 057301 (2004).

4. W. J. Marciano and A. I. Sanda, Phys. Lett. B 67, 303 (1977); B. W. Lee and R. E. Shrock, Phys. Rev. D 16, 1444 (1977); K. Fujikawa and R. Shrock, Phys. Rev. Lett. 45, 963 (1980).

5. J. F. Beacom and P. Vogel, Phys. Rev. Lett. 83, 5222 (1999); D. W. Liu et al., Phys. Rev. Lett. 93, 021802 (2004).

6. Z. Daraktchieva et al., Phys. Lett. B 615, 153 (2005); B. Xin et al., Phys. Rev. D 72, 012006 (2005).

7. G. G. Raffelt, Phys. Rept. 320, 319 (1999).

8. M. B. Voloshin, Sov. J. Nucl. Phys. 48, 512 (1988); S. M. Barr et al., Phys. Rev. Lett. 65, 2626 (1990).

9. S. Davidson, M. Gorbahn and A. Santamaria, Phys. Lett. B 626, 151 (2005). 